Results Out of the 235 patients that were reviewed, 71 patients either received thrombolysis and/or were sent for endovascular treatment, and 164 patients were not suitable for hyperacute treatment. Using the triage tool, we identified that $26 \%(n=61)$ of the rapid clinical assessment and 32\% ( $n=42)$ of CT perfusion scans performed could have been avoided. Conclusion Use of a web-based triage tool is not only effective to identify patients suitable for hyperacute management but also to avoid over-investigation and prioritize rapid neurological clinical assessments.

\section{RECURRENT HEADACHES WITH PSYCHOSIS, CSF LYMPHOCYTOSIS, VESSEL BEADING AND PAPILLOEDEMA- AUTOIMMUNE/VIRAL ENCEPHALITIS WITH VASCULOPATHY OR UNUSUAL PRESENTATION OF REVERSIBLE CEREBRAL VASOCONSTRICTION SYNDROME (RCVS)?}

${ }^{1}$ Christopher J Coppin ${ }^{*},{ }^{2}$ Michael Barnett, ${ }^{3}$ Lynette Masters, ${ }^{4}$ Amjed Aziz, ${ }^{5}$ Manisha Narasimhan*. 'Basic Physician Trainee, St George and Sutherland Hospitals, Sydney, NSW, Australia; ${ }^{2}$ Department of Neurology, Royal Prince Alfred Hospital; Brain and Mind Centre (USyd), Sydney, NSW, Australia; ${ }^{3}$ Neuroradiologist, IMED Radiology, Sydney, NSW, Australia; ${ }^{4}$ Department of Critical Care Medicine, Sutherland Hospital, Sydney, NSW, Australia; ${ }^{5}$ Department of Neurology, Sutherland Hospital, Sydney, NSW, Australia

\subsection{6/jnnp-2019-anzan.98}

Introduction Headache is a common Neurology presentation in both outpatient and ED settings. We present a challenging headache in a previously asymptomatic young female patient. Case A 27 year old female patient presented to ED with severe headache, vomiting and photophobia, after multiple presentations elsewhere, diagnosed as migraine, with normal neurological examination and MRI. Episodes of excruciating headache were associated with writhing and vomiting but spontaneously resolved with residual background pain. Indomethacin helped but she re-presented with headache and psychosis requiring intubation and ICU admission.

Investigations included CSF - protein 0.85 , leucocytosis 58 cells (lymphocytic) and normal cerebral venography. Acyclovir was commenced but viral PCRs were negative and she was extubated. MRI was suspicious for subtle posterior sulcal hyperintensity and beading in occipital and posterior cerebral arteries.

Another episode occurred ten days later requiring intubation, examination showed papilloedema. CSF examination showed raised pressure, normal protein and 48 lymphocytes; flow cytometry and cytology were unrevealing. NMDA receptor antibody returned positive in serum but not CSF. Subtle beading in right PCA branches remained. IVIG and steroids were used with dramatic response. Further investigation revealed positive EBV serology only and papilloedema resolved. Steroids were weaned and she remains in remission.

Conclusion We present an interesting case of recurrent acute headaches with intracranial hypertension, psychosis and CSF lymphocytosis. The recurrent headaches and vessel beading suggest RCVS, perhaps triggered by viral or autoimmune encephalitis. The NMDA receptor antibody result should be interpreted with caution given the absence of antibody in CSF and dramatic recovery.

\section{AXONAL POLYNEUROPATHY WITH ONSET IN YOUNG ADULTHOOD DUE TO TUBB3 MUTATION}

Po Sheng Yang* ${ }^{*}$ Kimberley K Forrest, Ian B Wilson. Neurology, Cairns Base Hospital, Cairns, QLD, Australia

\subsection{6/jnnp-2019-anzan.99}

Introduction The TUBB3 gene encodes the protein Beta-tubulin isotype III, a component of the microtubule cytoskeleton. Mutations in this gene have been associated with axonal polyneuropathy, however usually associated with congenital fibrosis of the extraocular muscles (CFOEM) and other abnormalities of cerebral development. ${ }^{1} 2$ We report a case of isolated neuropathy associated with a TUBB3 mutation.

Methods Case report - clinical information and next generation sequencing results were obtained.

Results A 64 year old man presented with a severe, progressive, length dependent sensorimotor polyneuropathy which commenced in his late twenties. There was no clinical involvement of the extraocular muscles and cognition was normal. Family history was limited, but there were no other members affected.

The patient had previously been extensively investigated including sural nerve biopsy, which confirmed axonal neuropathy without a specific diagnosis. Intravenous immunoglobulins and steroids had been trialled without benefit.

A neuromuscular gene panel utilising next generation sequencing was performed and demonstrated heterozygosity for a variant of the TUBB3 gene (D417N substitution).

Case series describing TUBB3 mutations show a large heterogeneity in phenotypic expression depending on the amino acid substitution. ${ }^{2-4}$ There is also heterogeneity in patients with $\mathrm{D} 417 \mathrm{~N}$ mutations, although a small number have been reported to develop a polyneuropathy without CFOEM $^{1}$.

Conclusions This case strengthens previous reports that TUBB3 mutation can be associated with a pure, axonal, sensorimotor polyneuropathy and highlights the use of next generation sequencing in streamlining the diagnostic process.

\section{REFERENCE}

1. Tischfield $\mathrm{M}$, et al. Human TUBB3 mutations perturb microtubule dynamics, kinesin interactions, and axon guidance. Cell 2010;140(1):pp.74-87.

2. Ncbi.nlm.nih.gov. (2019). Congenital fibrosis of the extraocular muscles - Conditions - GTR - NCBI. [online] Available at: https://www.ncbi.nlm.nih.gov/gtr/conditions/CN043677/ [Accessed 14 Feb. 2019].

3. Omim.org. (2019). OMIM Entry - *602661 - TUBULIN, BETA-3; TUBB3. [online] Available at: https://www.omim.org/entry/602661 [Accessed 14 Feb. 2019].

4. Krupa, K. and Bekiesinska-Figatowska, M. (2013). Congenital and Acquired Abnormalities of the Corpus Callosum: A Pictorial Essay. BioMed Research International, 2013, pp.1-14.

\section{A CASE OF SUSPECTED AUTOIMMUNE ENCEPHALITIS SECONDARY TO NIVOLUMAB}

${ }^{1}$ Jaimie Ho*, ${ }^{2}$ Yun Hwang, ${ }^{3}$ Martin Tio, ${ }^{3}$ Georgina Long, ${ }^{4}$ Eva Zhang. ${ }^{1}$ Gosford Hospital, Gosford, NSW, Australia; ${ }^{2}$ Neurology, Gosford Hospital, Gosford, NSW, Australia; ${ }^{3}$ Melanoma Institute Australia, Sydney, NSW, Australia; ${ }^{4}$ Royal North Shore Hospital, Sydney, NSW, Australia

\subsection{6/jnnp-2019-anzan. 100}

Objective To describe a case of suspected immune encephalitis following nivolumab for metastatic melanoma. 
Methods Retrospective review of clinical records and diagnostic tests.

Results A 78-year-old man with cerebral and lung melanoma metastases was treated with nivolumab, the newly developed monoclonal antibody which inhibits PD-1 deactivation of Tcells. He showed good radiological response.

Three months after ceasing nivolumab, he presented to ED with acute confusion, aphasia, left-sided weakness and neglect as a stroke call. MRI demonstrated no evidence of stroke or acute encephalitis. EEG showed diffuse slowing. Lumbar puncture was not performed as he was therapeutically anticoagulated and had begun to improve on empirical antibiotics and acyclovir. One week later, he suddenly deteriorated with fevers and reduced level of consciousness. Acyclovir was restarted. Repeat EEG revealed periodic epileptic discharges over the right hemisphere and MRI demonstrated hyperintense signal and diffusion restriction of the right mesial temporal lobe suggestive of encephalitis. Subsequent lumbar puncture was negative for HSV, VZV, enterovirus, and autoimmune encephalitis antibodies.

The patient's oncology team had been in support of a course of methylprednisone on suspicion of autoimmune encephalitis, but by the time the lumbar puncture results returned he was again improving. A diagnosis of suspected nivolumabinduced encephalitis was made based on exclusion of other possible diagnoses.

Conclusions The rising use of immune checkpoint inhibitors heralds an array of new and unexpected neurological complications including autoimmune encephalitis. A nationwide registry of possible cases may be helpful in improving our currently limited understanding of these emerging complications.

\section{COGNITIVE IMPAIRMENT IN THE COMMUNITY: A 16- MONTH AUDIT STUDY OF A NEUROLOGY MEMORY CLINIC IN THE INNER SYDNEY REGION}

${ }^{1}$ Louise Rigney, ${ }^{2}$ Lily Chen, ${ }^{3}$ Alexis Selby, ${ }^{4}$ Tejas Patel, ${ }^{5}$ Yun T Hwang, ${ }^{2}$ Anthony ED Mobbs, ${ }^{2,6,7}$ Rowena EA Mobbs*. 'Department of Neurology, Campbelltown Hospital, Campbelltown, NSW, Australia, Australia; ' 2 Sydney Cognitive, North Sydney, NSW, Australia; ${ }^{3}$ Department of Neurology, Northern Beaches Hospital, Frenchs Forest, NSW, Australia; ${ }^{4}$ Department of Neurology, Bankstown Hospital, Bankstown, NSW, Australia; ${ }^{5}$ Brain and Mind Centre, University of Sydney, Camperdown, NSW, Australia; ${ }^{6}$ University of Sydney, Camperdown, NSW, Australia; ${ }^{7}$ Macquarie University, Macquarie, NSW, Australia

10.1136/jnnp-2019-anzan.101

Introduction Neurodegenerative disease is increasingly common, but neurology service utilisation by this population is unclear. This study presents an integrated metropolitan neurology clinic as a model of care, and examines care pathways and characteristics in patients with cognitive decline.

Methods An audit of patients with dementia ( $n=136,51-96$ yr,M:F1.1:1) and mild cognitive impairment (MCI) $(n=28,56-$ 83 yr,M:F0.6:1) over a 16 month period was performed using retrospective analysis of demographic characteristics, clinical information and frailty measures.

Results The average duration of cognitive decline at presentation was 2.7 years in dementia $(\mathrm{n}=135, \mathrm{M}=3.1, \mathrm{~F}=2.3)$ and 1.5 years MCI $(\mathrm{n}=28, \mathrm{M}=1.7, \mathrm{~F}=1.3)$. Average age of patients with newly diagnosed dementia was 72 (M:F0.96:1) and MCI 70 (M:F1.03:1) with Alzheimer's disease in 86(64\%) dementia with Lewy bodies $13(10 \%)$ frontotemporal dementia $11(8 \%)$ and distinct overlap syndromes in $7(5 \%)$.
Depression was associated with younger age at diagnosis (ave.60) versus anxiety(74) and agitation(75). Psychosis was in 14(10\%) REM sleep behaviour disorder 6(5\%). In the 12 months prior, falls were seen in $30(22 \%)$ and weight loss 29 (21\%). Allied health input occurred in $81 \%$ of dementia versus $68 \%$ MCI cases.

Conclusions Delay in diagnosis from first clinical manifestation, high rate of psychological and medical comorbidity, presence of overlap syndromes, and allied health requirement, support the need for integrated neurological services in dementia.

\section{IS MBS RESTRICTION ON EEGS TRULY BENEFICIAL? RETROSPECTIVE OBSERVATIONAL ANALYSIS OF EEG REFERRAL PATTERNS}

${ }^{1}$ Chathri Ratnayake*, ${ }^{1}$ Ramja Kokulan, ${ }^{1,2}$ Patrick Carney. 'Department of Neurology, Eastern Health, Box Hill, VIC, Australia; ${ }^{2}$ Eastern Health Clinical School, Faculty of Medicine Dentistry and Health Sciences, Monash University, Box Hill, VIC, Australia

\subsection{6/jnnp-2019-anzan.102}

Introduction The November 2018 Medicare Benefits Schedule (MBS) Taskforce draft report recommends standardised national referral form for routine electroencephalogram (EEG) requests to prevent low value clinician referrals. MBS defines twelve clinical presentations where routine EEG is of relatively low diagnostic value. We aim to identify proportion of MBS defined low diagnostic value EEGs and likely referral patterns. Methods Retrospective single centre observational study was conducted from January to December 2018. All EEG referrals to a tertiary hospital neurodiagnostic unit were categorised as low or high yield based on MBS recommendation. Sub-group analysis of the low yield group was carried out.

Results Total of 1210 EEG referrals were analysed and 5 were excluded from analysis due to insufficient clinical data. Of these referrals 1114 (92.4\%) were for high yield indications, $77(6.4 \%)$ for low yield and $14(1.2 \%)$ were indeterminate as to low or high value. Of low yield referrals, $70 \%$ were referred for syncope or presyncope and 18.2\% for psychogenic nonepileptic seizures. Low yield EEGs were referred by hospital doctors, neurologists and general partitioners by proportions of $37.7 \%, 33.7 \%$ and $28.6 \%$ respectively. Four (5.2\%) low diagnostic value EEGs were abnormal.

Conclusion Current clinical practice for EEG referrals is in line with MBS draft review recommendations. We believe addition of a standardised referral form and restricting referrals will have minimal impact on referral quality.

\section{ALTERED PERIPHERAL AXONAL PROPERTIES IN FIBROMYALGIA}

${ }^{1,2,3}$ Cindy (Shin-YI) Lin*, ${ }^{4}$ Hao-Wen Teng, ${ }^{4,3}$ Jowy Tani, ${ }^{4}$ Hung-Ju Chen, ${ }^{4}{ }^{4} \mathrm{i}-$ Chen Lin, ${ }^{4}$ TsuiSan Chang, ${ }^{4}$ Jia-Ying Sung. 'University of Sydney, Sydney, NSW, Australia; ${ }^{2}$ Central Clinical School, Brain and Mind Centre, University of Sydney, Sydney, NSW, Australia; ${ }^{3}$ Neural Regenerative Medicine, College of Medical Science and Technology, Taipei Medical University, Taipei, Taiwan; ${ }^{4}$ Department of Neurology, Wan Fang Hospital, Taipei Medical University, Taipei, Taiwan

\subsection{6/jnnp-2019-anzan.103}

Introduction Fibromyalgia (FM), a common chronic widespread pain syndrome with neurological symptoms affecting $\sim 10 \%$ of the population worldwide. Whereas small fibre 